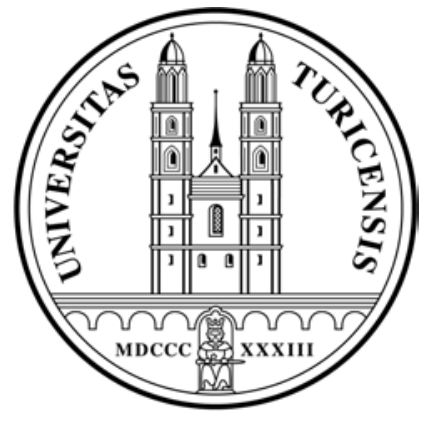

Institute for Empirical Research in Economics

University of Zurich

Working Paper Series

ISSN 1424-0459

Working Paper No. 255

Unique Equilibria in the Rubinstein Bargaining Model when the Payoff Set is Non-Convex

Wolfgang R. Köhler

October 2005 


\title{
Unique Equilibria in the Rubinstein Bargaining Model when the Payoff Set is Non-Convex*
}

\begin{abstract}
I give necessary and sufficient conditions for the uniqueness of the equilibrium in a wide class of Rubinstein bargaining models. The requirements encompass a class of non-convex or disconnected payoff sets with discontinuous Pareto frontiers. The equilibrium of the non-cooperative game is unique if the objective function of the corresponding Nash-bargaining game has a unique maximum. I extend the analysis to games where the time between offers is not constant.

Keywords: Bargaining

JEL Classification Number: C78
\end{abstract}

Wolfgang R. Köhler

IEW. University of Zürich, Winterthurerstrasse 30, 8006 Zürich, Switzerland

(E-mail: wolfgang.koehler@iew.unizh.ch)

*I would like to thank Thomas Jeitschko, Chris Laincz, Christoph Nitzsche, Huseyin Yildirim, and especially Curtis Taylor. 


\section{Introduction}

The standard full-information non-cooperative bargaining model is due to the seminal work of Rubinstein (1982). This model has been used widely in applications and has spawned a large body of theoretical literature. Despite the popularity and importance of the Rubinstein model, there have been few attempts to generalize the assumptions about the payoff set. I present a framework, which allows to generalize these assumptions, and state the necessary and sufficient conditions on the payoff set that guarantee that a unique equilibrium exists. The standard assumption is that the payoff set is convex. This assumption restricts applications since many bargaining situations involve non-convex payoff sets and players usually have no access to randomization devices so that they cannot propose lotteries.

Example 1 Consider the provision of a public good. For example, consider two institutions that organize a joint event and bargain about how much effort each has to put forth or spouses who bargain how to share the housework. Suppose that the public good yields a payoff of one, that the total effort that is necessary for provision is one, and that the disutility of effort $e_{i}$ for player $i$ is $\sqrt{e_{i}}$. The payoff of player $i$ is $p_{i}=1-\sqrt{e_{i}}$ with $e_{1}+e_{2}=1$. Note that efficiency requires that only one player contributes to the public good. The payoff set is non-convex and the Pareto-frontier is given by $p_{2}\left(p_{1}\right)=1-\sqrt{2 p_{1}-p_{1}^{2}}$ with $p_{1} \in[0,1]$.

Example 2 Non-convex payoff sets are common when players bargain over multi-dimensional issues and the payoff is not continuous in some dimensions. Consider two firms which use different technologies. Firms bargain about which technology is adopted as industry standard and about how to split the market. Payoff is linear in market shares $x$. If the technology of firm $i$ is adopted, firm i's payoff increases by 1 . For simplicity, suppose that the second technology is more efficient and that payoffs are $p_{1}=1+x_{1}$ and $p_{2}=x_{2}$ if the technology of firm 1 is adopted and $p_{1}=1.4 x_{1}$ and $p_{2}=1+1.4 x_{2}$ if the technology of firm 2 is adopted. The payoff set is non-convex and the Pareto-frontier is not continuous. 
Example 3 Consider a country that has defaulted and that now bargains with a bank about how much of the debt is forgiven and how much has to be repaid. For sovereign debt, collateral is usually negligible. Instead, the reputation of countries and banks affect the decision to default. If a bank has a reputation as a tough negotiator, countries have less incentives to default and the expected future payoff of the bank is higher. Let the country be player 1 and the bank be player 2. Suppose $p_{1}=-x$ and $p_{2}=x+f(x)$ where $x$ is the amount that is repaid and $f$ captures the effect of the bargaining outcome on the bank's reputation (i.e., on its future payoffs) with $f^{\prime}>0$. If the marginal effect on the reputation is increasing $\left(f^{\prime \prime}>0\right)$, the payoff set is non-convex.

These examples are not covered by regular bargaining theory because the payoff sets are nonconvex. Theorem 1(i) shows that these bargaining games have a unique equilibrium. Example 3 shows that the payoff set can be non-convex although players bargain over money. Other examples where the payoff set can be non-convex although side-payments are possible are players with interdependent preferences (e.g. Lopomo and Ok (2001)) or situations where money and the good in the original bargaining problem are strict complements.

While there exists an extensive literature that analyzes different offer structures (e.g., Perry and Reny (1993), Binmore (1987a)), bargaining over multiple issues (e.g., Inderst (2000) and literature cited therein) or when players can leave the negotiations (Ponsati and Sakovics (1998)), there have been few attempts to generalize conditions on the payoff set that ensure uniqueness. Binmore (1994) states a necessary condition for uniqueness of the equilibrium when the time between offers approaches zero. Binmore, Rubinstein, and Wolinsky (1986) show that for convex payoff sets the equilibrium of the Rubinstein game converges to the Nash-bargaining solution (NBS) as the time between offers approaches zero (see also Binmore (1987b)). There is no obvious way to extend the NBS to non-convex payoff sets. Conley and Wilkie (1996) use a geometric construction to define an extension of the NBS. Their extension selects a unique payoff combination that satisfies a desired set of axioms. Conley and Wilkie (1995) propose a non-cooperative game that implements their 
extension. Off the equilibrium path, their game makes use of lotteries. However, this extension is not supported by the equilibrium of the Rubinstein model. Herrero (1989) considers payoff sets with continuous and downward sloping Pareto frontiers and defines an extension of the NBS using an axiomatic characterization. Depending on the payoff set, this extension can take on a large number of values. As the time between offers approaches zero, the set of equilibria of the Rubinstein model converges to the set of extended Nash-solutions as defined by Herrero. I define the extension of the NBS as the set of payoff combinations that are the global maximizers of the weighted product of the payoffs. This extension is a subset of Herrero's solution. To avoid confusion, hereafter NBS refers to the set of global maximizers.

Section 2 introduces the model and summarizes the necessary and sufficient conditions for uniqueness of the equilibrium if the time between offers is constant. Section 3 states sufficient conditions for uniqueness when the time between offers is not constant. Section 4 discusses extensions of the model.

\section{Conditions for Uniqueness when the Time between Offers is Constant}

Two players bargain over a set $P \in R^{2}$ of feasible payoff combinations. Players alternate in proposing an allocation. The first $p \in P$ which is proposed by one player and accepted by the other is implemented and payoffs are realized. Player 1 starts and makes the first offer. Player 2 receives this offer at $\triangle t$. Player 2 chooses to either accept or reject the offer. If he accepts, the game is over and payoffs are realized; if he rejects, he makes a counteroffer to player 1. Player 1 receives this offer at $2 \triangle t$ and either accepts or makes a counteroffer and so on. Players discount future payoffs with interest rates $\tau_{1}, \tau_{2}>0$. Let $\delta_{i}$ be the discount factor of player $i$, with $\delta_{i}=e^{-\tau_{i} \triangle t}$. A player cannot be forced to agree, so players might disagree perpetually. In this case players receive a payoff of zero. As usual, equilibrium existence requires that a player accepts if he is indifferent. I 
assume that $P$ is closed and bounded and that there exists a $p \in P$ with $p \gg 0 .{ }^{1} \quad$ The maximum payoff which player $i$ can obtain is $\bar{p}_{i}=\max \left\{p_{i} \mid\left(p_{i}, p_{j}\right) \in P, p \geq 0\right\}$. For $p_{j} \in\left[0, \bar{p}_{j}\right]$ define $c_{i}$ with

$$
c_{i}\left(p_{j}\right)=\max \left\{p_{i} \mid\left(p_{i}, p_{j}^{\prime}\right) \in P \text { with } p_{j}^{\prime} \geq p_{j}\right\}
$$

The value $c_{i}\left(p_{j}\right)$ is the maximum payoff player $i$ can obtain if he offers at least $p_{j}$ to player $j$. The function $c_{i}$ is defined for all $p_{j} \in\left[0, \bar{p}_{j}\right]$. Note that it is possible that there exists $p_{j}$ such that $\left(c_{i}\left(p_{j}\right), p_{i}\right) \notin P$.

I use iterated conditional dominance to prove the uniqueness of the equilibrium. To do so, I define for each player $i$ acceptance and rejection levels $a_{i}^{n}$ and $r_{i}^{n}$ which depend on the round of elimination $n$. Let $a_{i}^{1}=\delta_{i} \bar{p}_{i}$ and $r_{i}^{1}=\delta_{i} c_{i}\left(a_{j}^{1}\right)$. For $n>1$, define $a_{i}^{n}$ and $r_{i}^{n}$ recursively with

$$
\begin{aligned}
a_{i}^{n} & =\delta_{i} c_{i}\left(\delta_{j} c_{j}\left(a_{i}^{n-1}\right)\right) \\
r_{i}^{n} & =\delta_{i} c_{i}\left(\delta_{j} c_{j}\left(r_{i}^{n-1}\right)\right)
\end{aligned}
$$

and let $a_{i}^{\infty}$ and $r_{i}^{\infty}$ be the limit of $\left(a_{i}^{n}\right)$ and $\left(r_{i}^{n}\right)$ as $n \rightarrow \infty$. Note that $\left(a_{i}^{\infty}\right)$ and $\left(r_{i}^{\infty}\right)$ are fixed points of $\delta_{i} c_{i}\left(\delta_{j} c_{j}\right)$.

Lemma 1 For player $i$ it is conditionally dominated to reject an offer that gives him a payoff greater than $a_{i}^{\infty}$ and to accept an offer that gives him a payoff less than $r_{i}^{\infty}$.

All proofs are relegated to the appendix.

Binmore et al. (1986) show for convex payoff sets that the equilibrium of the Rubinstein bargaining game converges to the NBS as the time between offers approaches zero. For convex payoff sets, the NBS is the payoff combination that maximizes the product of the payoffs. I extend this definition to non-convex payoff sets. While originally proposed as a symmetric solution, weights (which usually correspond to the players' interest rates) were introduced to capture the

\footnotetext{
1 The vector inequalities are represented by $\geq,>$, and $\gg$.
} 
bargaining power of the players. Let $\tau_{1}, \tau_{2}$ denote the weights and let $p_{1}^{N}, p_{2}^{N}$ denote the NBS where

$$
\left(p_{1}^{N}, p_{2}^{N}\right)=\underset{p \geq 0, p \in P}{\arg \max } p_{1}^{\tau_{2}} \cdot p_{2}^{\tau_{1}}
$$

Below, I define a collection $P_{\rho}$ of payoff sets such that whenever $P \in P_{\rho}$, the NBS is unique and the Rubinstein bargaining game has a unique equilibrium. Most of the bargaining literature imposes one of two restrictions: that the payoff set is convex (for non-cooperative bargaining) or $d$-comprehensive (for cooperative bargaining). I follow a different approach. Instead of using topological restictions, I define a collection of payoff sets in terms of $c_{1}$. Besides being the only tractable way of describing the collection of all payoff sets for which the equilibrium is unique, this approach has another advantage. In applications of bargaining theory it is not always straightforward to describe the payoff set. But $c_{1}$ is usually easy to compute because it is derived from a simple maximization problem.

Definition Given a bargaining game with constant time between offers and discount factors $\delta_{1}, \delta_{2}$, let $\rho=-\frac{\ln \delta_{2}}{\ln \delta_{1}}$. For closed and bounded payoff sets $P$ with $c_{i}$ right-continuous at zero, let $P_{\rho}$ be the collection of $P$ with

$$
P_{\rho}=\left\{P \mid c_{1}\left(p_{2}\right)^{-\rho} p_{2} \text { is strictly quasiconcave }\right\}
$$

Besides containing all convex payoff sets, the collection $P_{\rho}$ contains some non-convex payoff sets, disconnected payoff sets, and payoff sets with discontinuous Pareto frontiers. The quasiconcavity condition implies that $c_{1}$ is not too convex. In fact, $c_{1}$ being strictly $\rho$-concave is sufficient (but not necessary) to guarantee the uniqueness of the equilibrium.

Theorem 1 ( $\triangle t$ constant) (i) If $P \in P_{\rho}$, the payoff of player one is the same under all strategy profiles that survive iterated conditional dominance. If $P \in P_{\rho}$ and $c_{1}^{\prime+}\left(p_{2}\right)<0 \forall p_{2} \in$ $\left[\delta_{2} p_{2}^{N}, p_{2}^{N}\right)$, a unique strategy profile survives iterated conditional dominance. 
(ii) If $P \in P_{\rho}$, then as $\triangle t \rightarrow 0$ a unique strategy profile survives iterated conditional dominance and equilibrium payoffs are given by the Nash bargaining solution.

(iii) As $\triangle t \rightarrow 0$, a unique strategy profiles survives iterated conditional dominance if and only if $P \in P_{\rho}$.

Shaked and Sutton (1984) develop a simple method to compute the equilibrium in the Rubinstein bargaining game when the Pareto frontier is linear. When the SPNE is stationary, player 1 makes the same offer in every odd period. Let $M$ be the payoff of player 1 if 2 accepts. Therefore, 2 offers in every even period $\delta M$ to 1 and gets the remaining payoff. Hence, 1 offers in odd periods the discounted payoff 2 would get when 2's offer would be accepted. The remaining payoff for 1 if 2 accepts is equal to $M$. The equilibrium is unique whenever there is a unique $M$ that satisfies this relation which is true iff $\delta_{2} c_{2}\left(\delta_{1} c_{1}\right)$ has a unique fixed point. Since this proof relies on the stationarity of the equilibrium, it cannot be used when the time between offers is not constant even when the Pareto-frontier is linear.

\section{Uniqueness when the Time between Offers is not Constant}

In many circumstances under which people bargain, the time between offers is not constant. For example, consider boards that have to approve offers but that do not meet at night or on holidays. In applications, constant time between offers is a convenient assumption, especially if it allows to compute explicit payoffs. However, to justify this assumption, it is necessary to know under which conditions a unique equilibrium exists when the time between offers is not constant.

Let $\triangle_{t}$ be the lenght of period $t$, i.e. $\triangle_{t}$ is the difference between the time when the $t$-th offer is received and the time when it is made by the other player. Let $\delta_{i}^{t}$ be the corresponding discount factor with $\delta_{i}^{t}=e^{-\tau_{i} \triangle_{t}}$. When the time between offers is not constant, define for all $t, t+1$ with $t$ even the NBS:

$$
p_{2}^{N}=\underset{p \geq 0, p \in P}{\arg \max } c_{1}\left(p_{2}\right)^{\frac{\ln \delta_{2}^{t}}{\ln \delta_{1}^{t+1}}} \cdot p_{2} \text { and } p_{1}^{N}=c_{1}\left(p_{2}^{N}\right)
$$


For $t$ even, let $\underline{p}_{2}^{N}=\min \left\{\delta_{2}^{t} p_{2}^{N}\right\}$ and $\bar{p}_{2}^{N}=\max \left\{p_{2}^{N}\right\}$ and let $\underline{\rho}=\min \left\{-\frac{\ln \delta_{2}^{t}}{\ln \delta_{1}^{t+1}}\right\}$ and $\bar{\rho}=$ $\max \left\{-\frac{\ln \delta_{2}^{t}}{\ln \delta_{1}^{t+1}}\right\}$.

Theorem 2 ( $\triangle t$ not constant) Let $P \in P_{\rho} \forall \rho \in[\underline{\rho}, \bar{\rho}]$ and suppose there exists $\varepsilon>0$ such that $c_{1}$ is strictly $\bar{\rho}$-concave for all $p \in\left[\underline{p}_{2}^{N}-\varepsilon, \bar{p}_{2}^{N}+\varepsilon\right]$. Then there exists a unique strategy profile that survives iterated conditional dominance.

When the time between offers is not constant, it is possible to provide limits for the equilibrium payoffs but not to compute explicit equlibrium payoffs. As $\triangle t \rightarrow 0$ the equilibrium payoff of player 1 is in the interval $\left[\min _{t} p_{1}^{N}, \max _{t} p_{1}^{N}\right]$ and similar for player 2 .

\section{Generalizations}

In many situations, players receive some income flow or incur some cost during the bargaining (e.g. strike pay, cost of negotiations). Furthermore, players do not always know when the other player will receive the offer or when the other player will be able to respond. In this case, the time between offers is random. Additonally, in many situations it is possible that the bargaining opportunity disappears or that the bargaining process breaks down.

In this section, I generalize the Rubinstein bargaining model to account for these possibilities. The analysis of bargaining games with income flows and the possibility of a breakdown is not new (see Muthoo (1999) for a detailed overview). When the time between offers is constant, one can use the fact that the equilibrium is stationary and the computation of the equilibrium is essentially the same as when income flows and breakdown payoffs are zero. Instead of following this approach, I develop a new technique. Specifically, I show that income flows and breakdown payoffs can be normalized to zero. That means that there exists a bargaining game without income flows or breakdown payoffs that is equivalent (in terms of strategies and payoffs) to the original game. This 
technique allows to analyze bargaining games with income flows and breakdown payoffs when the time between offers is not constant.

Except for the additional features, the model is the same as the alternating offer model presented above. Player 1 starts by making the first offer at time $t_{1}$. Player 2 receives this offer at $t_{1}+\zeta_{1}$, where $\zeta$ is the realization of a random variable which captures delays in the transmission of the offer. At $t_{2}=t_{1}+\zeta_{1}$ player 2 chooses to either accept or reject the offer. If he rejects, he makes a counteroffer, where player 1 receives this offer at $t_{2}+\zeta_{2}$ and so on. The delay $\zeta_{k}$ is drawn from the distribution function $H_{k}$ with density $h_{k}$. I assume that the expected value of $\zeta_{k}$ is positive. During one unit of time the bargaining process breaks down with probability $1-\mu$. In this case, both players get their breakdown payoffs $b_{1}$ and $b_{2}$. The expected payoff for player $i$ from the breakdown of the bargaining between $t_{k}$ and $t_{k}+\zeta_{k}$ is:

$$
b_{i}^{k}=\int_{0}^{\infty} \int_{0}^{t}-\ln \mu \cdot \mu^{z} b_{i} e^{-\tau_{i} z} d z h_{k}(t) d t
$$

where $\mu^{z}$ is the probability that the bargaining did not break down between time $t_{k}$ and $t_{k}+z$ and $-\ln \mu$ is the instantaneous probability of a breakdown. During the bargaining process, players receive an income flow $\gamma_{1}$ and $\gamma_{2}$. The expected payoff of player $i$ from the income flow between $t_{k}$ and $t_{k}+\zeta_{k}$ discounted to $t_{k}$ is:

$$
\gamma_{i}^{k}=\int_{0}^{\infty} \mu^{t} \gamma_{i} e^{-\tau_{i} t}\left(1-H_{k}(t)\right) d t
$$

The expected payoff of a player is the sum of his expected payoffs from the income flow, the breakdown payoff and the implemented offer $p$. If players disagree forever, they receive $\gamma$ as long as the bargaining process does not break down plus $b$ when the bargaining breaks down. Let $s_{i}$ denote the expected disagreement payoff of player $i$ :

$$
s_{i}=\frac{\gamma_{i}-\ln \mu \cdot b_{i}}{\tau_{i}-\ln \mu}
$$

I assume that there exists a $p \in P$ with $p \gg s$. If player $i$ offers $p$ at time $t_{s}$ to player $j$ the expression "the payoff of $j$ from $p$ " refers to the payoff player $j$ gets at $t_{s+1}$ from accepting $p$ but 
does not include earlier income flows. For $p_{j} \in\left[s_{j}, \bar{p}_{j}\right]$ define $c_{i}$ with $c_{i}\left(p_{j}\right)=\max \left\{p_{i} \mid\left(p_{i}, p_{j}^{\prime}\right) \in P\right.$ with $p_{j}^{\prime} \geq p_{j}$. Let $\delta_{i}^{k}$ be discounted value of an offer of 1 in period $k$ when income flow and breakdown payoffs are not taken into account, i.e. $\delta_{i}^{k}=\int_{0}^{\infty} \mu^{t} e^{-\tau_{i} t} h_{k}(t) d t$. The discount factor consists of two terms, the usual discounting term $e^{-\tau t}$, and the probability $\mu^{t}$ that the bargaining process does not break down before $t$. The discount factor captures the two motives to reach an agreement: impatience and the fear that the bargaining opportunity might disappear. Similar to the case when breakdown payoffs and income flows are zero, I define acceptance and rejection levels which depend on the round of elimination:

$$
\begin{aligned}
& a_{i}^{n}=\delta_{i}^{1} c_{i}\left(\delta_{j}^{2} c_{j}\left(a_{i}^{n-1}\right)+\gamma_{j}^{2}+b_{j}^{2}\right)+\gamma_{i}^{1}+b_{i}^{1} \\
& r_{i}^{n}=\delta_{i}^{1} c_{i}\left(\delta_{j}^{2} c_{j}\left(r_{i}^{n-1}\right)+\gamma_{j}^{2}+b_{j}^{2}\right)+\gamma_{i}^{1}+b_{i}^{1}
\end{aligned}
$$

When income flows or breakdown payoffs are non-zero, the expressions for $a_{i}^{n}, r_{i}^{n}$ are cumbersome for proving uniqueness. To normalize $\gamma$ and $b$, let $P^{s}=\left\{\left(p_{i}-s_{i}, p_{j}-s_{j}\right) \mid p \in P\right\}$ and define

$$
\begin{aligned}
& c_{i}^{s}\left(u_{j}\right)=c_{i}\left(u_{j}+s_{j}\right)-s_{i} \\
& c_{j}^{s}\left(u_{i}\right)=c_{j}\left(u_{i}+s_{i}\right)-s_{j}
\end{aligned}
$$

where the superscript $s$ indicates the normalized expessions. Note that $c_{j}^{s}:\left[0, \bar{p}_{i}-s_{i}\right] \rightarrow\left[0, \bar{p}_{j}-s_{j}\right]$ and similar for $c_{i}^{s}$. For a given sequence of distribution functions $H_{k}$ and given interest rates $\tau_{1}$ and $\tau_{2}$, let $\Gamma(P, \gamma, b)$ be the bargaining game with payoff set $P$, income flow $\gamma$, and breakdown payoff $b$. Strategies can be described by a cut-off payoff such that a player accepts or rejects an offer and by the payoff that a player offers to the other player. Given $\Gamma(P, \gamma, b)$, let $f(\Gamma(P, \gamma, b))$ be the set of strategies that survive iterated conditional dominance. Lemma 2 shows that it is possible to normalize income flows and breakdown payoffs to zero and that it is sufficient to prove uniqueness and to compute equilibrium strategies for the simpler game where $\gamma=b=0$.

Lemma 2 If $\triangle t$ is constant and $P^{s} \in P_{\rho}$, then $f(\Gamma(P, \gamma, b))=f\left(\Gamma\left(P^{s}, 0,0\right)\right)+s$. For $\triangle t$ not constant, if $P^{s} \in P_{\rho} \forall \rho \in[\underline{\rho}, \bar{\rho}]$ and if there exists $\varepsilon>0$ such that $c_{1}$ is strictly $\bar{\rho}$-concave for all 
$p \in\left[\underline{p}_{2}^{N}-\varepsilon, \bar{p}_{2}^{N}+\varepsilon\right]$, then $f(\Gamma(P, \gamma, b))=f\left(\Gamma\left(P^{s}, 0,0\right)\right)+s$.

Note that payoff set and the functions $c_{1}, c_{2}$ are normalized but that discount factors in $\Gamma\left(P^{s}, 0,0\right)$ are the same as in $\Gamma(P, \gamma, b)$.

\section{Conclusions}

This paper generalizes the assumptions about the payoff set in the Rubinstein bargaining model and gives necessary and sufficient conditions for uniqueness. The normalization technique makes it possible to analyze games with income flows and breakdowns when the time between offers is not constant. This is technique should also prove useful for other questions since it allows to analyze bargaining games where the payoff set changes over time.

\section{Appendix}

Lemma 1 For player $i$ it is conditionally dominated to reject an offer that gives him a payoff greater than $a_{i}^{\infty}$ and to accept an offer that gives him a payoff less than $r_{i}^{\infty}$.

Proof. Since zero is the minimax payoff, it is strictly dominated to accept an offer which yields less than zero. Player 1 makes the first offer. The discounted maximum payoff that player 2 can obtain when he rejects the offer is $\delta_{2} \bar{p}_{2}$. Hence, for player 2 it is conditionally dominated to reject an offer where $p_{2}>a_{2}^{1}=\delta_{2} \bar{p}_{2}$ where the superscript in $a_{2}^{1}$ refers to the first round of elimination. Similar, it is conditionally dominated for player 2 to accept an offer which yields him less than $r_{2}^{1}=\delta_{2} c_{2}\left(a_{1}^{1}\right)$, because this is the amount he can gain for sure by offering $a_{1}^{1}$ in the next period to player 1 . Therefore, player 1 will offer at least $r_{2}^{1}$. By assumption $\bar{p}_{i}>0$ so that $a_{i}^{1}<\bar{p}_{i}$. But then $r_{i}^{1} \geq 0$. Similarly, if player 2 rejects the offer of player 1 he would have to offer at least $r_{1}^{1}$ at time $t_{2}$. Therefore, it is conditionally dominated for player 2 to reject an offer where $p_{2}>a_{2}^{2}=\delta_{2} c_{2}\left(r_{1}^{1}\right)$ where $\delta_{2} c_{2}\left(r_{1}^{1}\right)=\delta_{2} c_{2}\left(\delta_{1} c_{1}\left(a_{2}^{1}\right)\right)$, and so on. For $n>1$, the expressions for 
$a_{i}^{n}$ and $r_{i}^{n}$ can be written recursively with $a_{i}^{n}=\delta_{i} c_{i}\left(\delta_{j} c_{j}\left(a_{i}^{n-1}\right)\right)$ and $r_{i}^{n}=\delta_{i} c_{i}\left(\delta_{j} c_{j}\left(r_{i}^{n-1}\right)\right)$. Note that $a_{i}^{1} \geq r_{i}^{1}$. Since $c_{j}$ is weakly decreasing we have $a_{j}^{2} \geq r_{j}^{2}$. From induction follows that $a_{i}^{n} \geq r_{i}^{n}$ for all $n>0$. Since $\left(a_{i}^{n}\right)$ and $\left(r_{i}^{n}\right)$ are monotone, we have $a_{i}^{n} \geq r_{i}^{k} \forall n, k$. The sequences $\left(a_{i}^{n}\right)$ and $\left(r_{i}^{n}\right)$ are monotone and their range is a subset of $\left[0, \bar{p}_{i}\right]$. Therefore, $\left(a_{i}^{n}\right)$ and $\left(r_{i}^{n}\right)$ converge and the limits $a_{i}^{\infty}$ and $r_{i}^{\infty}$ exist. Hence, for player $i$ it is conditionally dominated to reject an offer that yields more $a_{i}^{\infty}$ or to reject an offer that yields less than $r_{i}^{\infty}$.

Before I prove Theorem 1, I introduce two definitions that are used in the proofs. To show that the function $\delta_{1} c_{1}\left(\delta_{2} c_{2}\right)$ has a unique fixed point and that $\left(a_{2}^{n}\right)$ and $\left(r_{2}^{n}\right)$ converge to the fixed point, I define a function $v$ such that every point in the domain of $v$ is a fixed point of $\delta_{1} v^{-1}\left(\delta_{2} v\right)$. The function $v$ is exactly $\rho$-concave with $\rho=-\frac{\ln \delta_{2}}{\ln \delta_{1}}$.

Definition Let $x_{\lambda}=\lambda x_{1}+(1-\lambda) x_{2}$. A positive-valued function $g$ with convex domain $X$ is $\rho$-concave for $\rho \neq 0$ and $|\rho|<\infty$ if $\forall x_{1}, x_{2} \in X$ and $\lambda \in(0,1)$

$$
g\left(x_{\lambda}\right) \geq\left(\lambda g\left(x_{1}\right)^{\rho}+(1-\lambda) g\left(x_{2}\right)^{\rho}\right)^{\frac{1}{\rho}}
$$

The function $g$ is strictly $\rho$-concave (exactly $\rho$-concave) if the inequality holds strictly (with equality).

Definition Given $0<\delta_{1}, \delta_{2}<1$, let $V_{\rho}$ be the family of all $v_{\alpha}: R_{+} \backslash\{0\} \rightarrow R_{+} \backslash\{0\}$ with $\alpha>0$ and

$$
v_{\alpha}(x)=\left(\frac{x}{\alpha}\right)^{-\frac{\ln \delta_{1}}{\ln \delta_{2}}}
$$

Note that $\delta_{2} v_{\alpha}^{-1}\left(\delta_{1} v_{\alpha}(x)\right)=x$, that $v_{\alpha}$ is exactly $\rho$-concave, and that $\left[v_{\alpha}(x)\right]^{-\rho} \cdot x=\alpha$.

Theorem 1 ( $\triangle t$ constant) (i) If $P \in P_{\rho}$, the payoff of player one is the same under all strategy profiles that survive iterated conditional dominance. If $P \in P_{\rho}$ and $c_{1}^{\prime+}\left(p_{2}\right)<0 \forall p_{2} \in$ $\left[\delta_{2} p_{2}^{N}, p_{2}^{N}\right)$, a unique strategy profile survives iterated conditional dominance. 
(ii) If $P \in P_{\rho}$, then as $\triangle t \rightarrow 0$ a unique strategy profile survives iterated conditional dominance and equilibrium payoffs are given by the Nash bargaining solution.

(iii) As $\triangle t \rightarrow 0$, a unique strategy profiles survives iterated conditional dominance if and only if $P \in P_{\rho}$.

Proof. (i) The function $\delta_{2} c_{2}\left(\delta_{1} c_{1}\right):\left[0, \bar{p}_{2}\right] \longrightarrow\left[0, \bar{p}_{2}\right]$ is non-decreasing. From Tarski's fixed point theorem follows that $\delta_{2} c_{2}\left(\delta_{1} c_{1}\right)$ has at least one fixed point. If $P \in P_{\rho}$, the function $c_{1}\left(p_{2}\right)^{-\rho} p_{2}$ is strictly quasiconcave. Since $\left[v_{\alpha}(p)\right]^{-\rho} \cdot p=\alpha$, all $v_{\alpha} \in V_{\rho}$ intersect $c_{1}$ at most twice. Let $p_{f}$ be an arbitrary fixed point of $\delta_{2} c_{2}\left(\delta_{1} c_{1}\right)$. Note that $P \in P_{\rho}$ implies that $c_{i}$ is right-continuous at zero and, therefore, $c_{i}\left(\delta_{j} \bar{p}_{j}\right)>0$. Hence, $\delta_{2} c_{2}\left(\delta_{1} c_{1}(0)\right)>0$ and $p_{f}>0$. The range of $c_{2}$ is a subset of $\left[0, \bar{p}_{2}\right]$. Hence, $p_{f} \leq \delta_{2} \bar{p}_{2}$. Recall that $v_{\alpha}: R_{+} \backslash\{0\} \rightarrow R_{+} \backslash\{0\}$. Since $\delta_{2} v_{\alpha}^{-1}\left(\delta_{1} v_{\alpha}(p)\right)=p$ and $c_{1}\left(\delta_{2} \bar{p}_{2}\right)>0$ we know that for any fixed point $p_{f}$ of $\delta_{2} c_{2}\left(\delta_{1} c_{1}\right) \exists \alpha$ s.t. $v_{\alpha}\left(p_{f}\right)=c_{1}\left(p_{f}\right)$ and $v_{\alpha}\left(\frac{p_{f}}{\delta_{2}}\right)=\delta_{1} c_{1}\left(p_{f}\right)$. Strict quasiconcavity of $c_{1}\left(p_{2}\right)^{-\rho} p_{2}$ implies that $v_{\alpha}(p)<c_{1}(p) \forall p \in\left(p_{f}, \frac{p_{f}}{\delta_{2}}\right)$. Let $\bar{\alpha}=\max \left\{\alpha \mid \exists p\right.$ with $\left.c_{1}(p)=v_{\alpha}(p)\right\}$. Since $c_{1}\left(p_{2}\right)^{-\rho} p_{2}$ is strictly quasiconcave and since $\left[v_{\alpha}(p)\right]^{-\rho} \cdot p=\alpha$, there exists a unique $\widehat{p}$ such that $c_{1}(\widehat{p})=v_{\bar{\alpha}}(\widehat{p})$ with $c_{1}(p)<v_{\bar{\alpha}}(p) \forall p \neq \widehat{p}$. Thus $\widehat{p}=\arg \max c_{1}\left(p_{2}\right)^{-\rho} p_{2}$. From the definition of NBS follows $\widehat{p}=p_{2}^{N}$.

For an arbitrary $\alpha<\bar{\alpha}$, let $p_{a}=\min \left\{p \mid v_{\alpha}\left(p_{a}\right)=c_{1}\left(p_{a}\right)\right\}$. By construction, $c_{1}$ is weakly decreasing. Therefore, $P \in P_{\rho}$ implies that $c_{1}$ is continuous for $p<\widehat{p}$. Since $c_{1}(0)=\bar{p}_{1}$ and $\lim _{p \rightarrow 0} v_{\alpha}(p)=\infty$, for every $\alpha \in(0, \bar{\alpha})$ exists a unique $p_{a}$ with $p_{a}<\widehat{p}$. Given $\alpha$, let $p_{b}$ denote the unique $p \geq \widehat{p}$ s.t. $c_{1}^{-}\left(p_{b}\right) \geq v_{\alpha}\left(p_{b}\right) \geq c_{1}^{+}\left(p_{b}\right)$. Since $P$ is closed, from the definition of $c_{1}$ follows that $c_{1}^{-}\left(p_{b}\right)=c_{1}\left(p_{b}\right)$. Note that $v_{\alpha}(p)<c_{1}(p)$ for $p \in\left(p_{a}, p_{b}\right)$ and $v_{\alpha}(p)>c_{1}(p)$ for $p \notin\left[p_{a}, p_{b}\right]$.

Strict quasiconcavity of $c_{1}\left(p_{2}\right)^{-\rho} p_{2}$ implies that $p_{a}$ is strictly increasing in $\alpha$ while $p_{b}$ is weakly decreasing. Hence there exists a unique $\alpha_{f}$ such that for $p_{a}$ and $p_{b}$ that are defined by $\alpha_{f}$ it is true that $p_{a}=\delta_{2} p_{b}$. I show next that the $p_{a}$ that is defined by $\alpha_{f}$ is the unique fixed point of $\delta_{2} c_{2}\left(\delta_{1} c_{1}\right)$.

Let $\widetilde{p}_{f}$ and $\frac{\widetilde{p}_{f}}{\delta_{2}}$ denote the unique $p_{a}$ and $p_{b}$ where $p_{a}=\delta_{2} p_{b}$. From $\delta_{2} v_{\alpha}^{-1}\left(\delta_{1} v_{\alpha}(p)\right)=p$ follows 
that $\delta_{1} v_{\alpha}\left(\widetilde{p}_{f}\right)=v_{\alpha}\left(\frac{\widetilde{p}_{f}}{\delta_{2}}\right)$. From $v_{\alpha_{f}}\left(\widetilde{p}_{f}\right)=c_{1}\left(\widetilde{p}_{f}\right)$ follows that $\delta_{1} c_{1}\left(\widetilde{p}_{f}\right)=v_{\alpha_{f}}\left(\frac{\widetilde{p}_{f}}{\delta_{2}}\right)$. Recall that $c_{1}\left(p_{2}\right)^{-\rho} p_{2}$ is strictly quasiconcave and that $p_{b} \geq \widehat{p}$, i.e. $\frac{\widetilde{p}_{f}}{\delta_{2}} \geq \widehat{p}$. There are two cases:

Case I: $c_{1}\left(\frac{\widetilde{p}_{f}}{\delta_{2}}\right)=v_{\alpha_{f}}\left(\frac{\widetilde{p}_{f}}{\delta_{2}}\right)$ and $c_{1}^{\prime}\left(\frac{\widetilde{p}_{f}}{\delta_{2}}\right)<0$.

Since $c_{1}^{\prime}\left(\frac{\widetilde{p}_{f}}{\delta_{2}}\right)<0$ there exists no $p_{2}>\frac{\widetilde{p}_{f}}{\delta_{2}}$ and $p_{1} \geq c_{1}\left(\frac{\widetilde{p}_{f}}{\delta_{2}}\right)$ with $\left(p_{1}, p_{2}\right) \in P$. Thus $\delta_{1} c_{1}\left(\widetilde{p}_{f}\right)=$ $v_{\alpha_{f}}\left(\frac{\widetilde{p}_{f}}{\delta_{2}}\right), c_{1}\left(\frac{\widetilde{p}_{f}}{\delta_{2}}\right)=v_{\alpha_{f}}\left(\frac{\widetilde{p}_{f}}{\delta_{2}}\right)$, and $c_{1}^{\prime}\left(\frac{\widetilde{p}_{f}}{\delta_{2}}\right)<0$ imply that $c_{2}\left(\delta_{1} c_{1}\left(\widetilde{p}_{f}\right)\right)=v_{\alpha}^{-1}\left(\delta_{1} v_{\alpha}\left(\widetilde{p}_{f}\right)\right)=\frac{\widetilde{p}_{f}}{\delta_{2}}$.

Case II: $c_{1}\left(\frac{\widetilde{p}_{f}}{\delta_{2}}\right) \geq v_{\alpha_{f}}\left(\frac{\widetilde{p}_{f}}{\delta_{2}}\right) \geq c_{1}^{+}\left(\frac{\widetilde{p}_{f}}{\delta_{2}}\right)$.

Strict quasiconcavity of $c_{1}\left(p_{2}\right)^{-\rho} p_{2}$ implies that $c_{1}$ is strictly decreasing for all $p \geq \widehat{p}$. Since $\frac{\widetilde{p}_{f}}{\delta_{2}} \geq \widehat{p}$, there exists no $p_{2}>\frac{\widetilde{p}_{f}}{\delta_{2}}$ and $p_{1} \geq c_{1}^{+}\left(\frac{\widetilde{p}_{f}}{\delta_{2}}\right)$ such that $\left(p_{1}, p_{2}\right) \in P$. Hence, for all $p \in\left[c_{1}^{+}\left(\frac{\widetilde{p}_{f}}{\delta_{2}}\right), c_{1}\left(\frac{\widetilde{p}_{f}}{\delta_{2}}\right)\right]$ we have $c_{2}(p)=\frac{\widetilde{p}_{f}}{\delta_{2}}$. Thus $\delta_{1} c_{1}\left(\widetilde{p}_{f}\right)=v_{\alpha_{f}}\left(\frac{\widetilde{p}_{f}}{\delta_{2}}\right) \operatorname{implies} c_{2}\left(\delta_{1} c_{1}\left(\widetilde{p}_{f}\right)\right)=\frac{\widetilde{p}_{f}}{\delta_{2}}$.

In both cases $\delta_{2} c_{2}\left(\delta_{1} c_{1}\left(\widetilde{p}_{f}\right)\right)=\widetilde{p}_{f}$ and, therefore, $\widetilde{p}_{f}=p_{f}$, i.e., $\widetilde{p}_{f}$ is the unique fixed point of $\delta_{2} c_{2}\left(\delta_{1} c_{1}\right)$. From $p_{f}<\widehat{p} \leq \frac{p_{f}}{\delta_{2}}$ and $\widehat{p}=p_{2}^{N}$ follows that $p_{f} \in\left[\delta_{2} p_{2}^{N}, p_{2}^{N}\right)$. Since $\delta_{2} c_{2}\left(\delta_{1} c_{1}\right)$ is non-decreasing and has a unique fixed point $p_{f}$ we have $\delta_{2} c_{2}\left(\delta_{1} c_{1}(p)\right)>p$ for $0 \leq p<p_{f}$ and $\delta_{2} c_{2}\left(\delta_{1} c_{1}(p)\right)<p$ for $p_{f}<p \leq \bar{p}_{2}$. Hence $\left(r_{2}^{n}\right)$ and $\left(a_{2}^{n}\right)$ converge to $p_{f}$. Note that $r_{1}^{n}=\delta_{1} c_{1}\left(a_{2}^{n}\right)$ and $a_{1}^{n}=\delta_{1} c_{1}\left(r_{2}^{n-1}\right)$. Hence, $\left(r_{1}^{n}\right)$ and $\left(a_{1}^{n}\right)$ converge to $\delta_{1} c_{1}\left(p_{f}\right)$.

From Lemma 1 follows that it is conditionally dominated for player 2 to reject (accept) an offer where $p_{2}>p_{f}\left(p_{2}<p_{f}\right)$. Therefore, it is conditionally dominated for player 1 to accept (reject) an offer where $p_{1}<\delta_{1} c_{1}\left(p_{f}\right)\left(p_{1}>\delta_{1} c_{1}\left(p_{f}\right)\right)$. Hence, it is conditionally dominated for player 2 to offer $p_{1}<\delta_{1} c_{1}\left(p_{f}\right)$ or $p_{1}$ such that $c_{2}\left(p_{1}\right)<c_{2}\left(\delta_{1} c_{1}\left(p_{f}\right)\right)$. Additionally, it is conditionally dominated for player 1 to offer $p_{2}<p_{f}$ or $p_{2}$ such that $c_{1}\left(p_{2}\right)<c_{1}\left(p_{f}\right)$. Since $c_{i}$ is weakly decreasing, this implies that in all equilibria, the payoff of player 1 is $c_{1}\left(p_{f}\right)$. Since $p_{f} \in\left[\delta_{2} p_{2}^{N}, p_{2}^{N}\right)$, the condition that $c_{1}^{\prime+}<0 \forall p_{2} \in\left[\delta_{2} p_{2}^{N}, p_{2}^{N}\right)$ ensures that $c_{1}^{\prime+}\left(p_{f}\right)<0$, i.e. there is a trade-off between the payoff of player 1 and 2. Therefore, the equilibrium is unique if $c_{1}^{++}<0 \forall p_{2} \in\left[\delta_{2} p_{2}^{N}, p_{2}^{N}\right)$.

(ii) Convergence to NBS:

Note that $\rho$ does not depend on $\triangle t$. Let $p_{f}(\triangle t)$ be the fixed point of $\delta_{2} c_{2}\left(\delta_{1} c_{1}\right)$ as a function of $\triangle t$. Since $p_{f} \in\left[\delta_{2} p_{2}^{N}, p_{2}^{N}\right)$, we have $\lim _{\Delta t \rightarrow 0} p_{f}(\triangle t)=p_{2}^{N}$. Since $p_{2}^{N}$ is the unique maximizer of $c_{1}\left(p_{2}\right)^{-\rho} p_{2}$, either $c_{1}^{\prime}\left(p_{2}^{N}\right)<0$ or $c_{1}\left(p_{2}^{N}\right)>c_{1}^{+}\left(p_{2}^{N}\right)$. Hence, in the limit as $\Delta t \rightarrow 0$, there 
exists a unique $p=p_{2}^{N}$ such that $c_{1}(p) \geq c_{1}\left(p_{2}^{N}\right)$ and $p \geq p_{2}^{N}$. Therefore, as $\Delta t \rightarrow 0$, we have $r_{i}^{\infty}=a_{i}^{\infty}=p_{2}^{N}$ and from Lemma 1 follows that a unique strategy profile survives iterated conditional dominance and that equilibrium payoffs are given by the NBS.

(iii) In part (ii), I have shown that in the limit as $\Delta t \rightarrow 0$ the equilibrium converges to the NBS, i.e. that the equilibrium is unique. It remains to show that as $\Delta t \rightarrow 0$ at least two equilibria exist if $P \notin P_{\rho}$. The set of subgame perfect Nash-equilibria (SPNE) is a subset of the set of strategy profiles that survive iterated conditional dominance. Therefore, it is sufficient to show that at least two SPNE exist if $P \notin P_{\rho}$. I show first that at least two SPNE exist if $c_{i}$ is not right-continuous at zero and then that at least two SPNE exist if $c_{1}\left(p_{2}\right)^{-\rho} p_{2}$ is not strictly quasiconcave.

If $c_{i}$ is not right-continuous at zero, then $c_{i}(0)>c_{i}^{+}(0)$ where $c_{i}(0)=\bar{p}_{i}$. At least two SPNE exist. In the first SPNE, player $i$ offers 0 and rejects any offer that yields him less than $\bar{p}_{i}$. Player $j$ offers $\bar{p}_{i}$ and accepts any offer. In the second SPNE corresponds to the NBS.

If $c_{1}\left(p_{2}\right)^{-\rho} p_{2}$ is not strictly quasiconcave, there are two possible cases.

Case 1: There exists an $\alpha$ and an interval $I$ s.t. $c_{1}(p)=v_{\alpha}(p) \forall p \in I$.

Let $a=\inf I$ and $b=\sup I$. Since $v_{\alpha}$ is continuous and strictly decreasing, from $c_{1}(p)=v_{\alpha}(p)$ $\forall p \in I$ follows that $c_{1}$ is continuous and strictly decreasing on $(a, b]$. Hence $c_{2}$ is continuous on $\left(\delta_{1} c_{1}(b), \delta_{1} c_{1}(a)\right.$ ] (Note that this is true regardless of $c_{1}$ being continuous at $a$ ). Rewrite $c_{1}(p)=v_{\alpha}(p)$ as $c_{1}(a+\varepsilon)=v_{\alpha}(a+\varepsilon) \forall \varepsilon \in(0, b-a]$. Define $\overline{\triangle t}$ such that $a=b \cdot e^{-\tau_{2} \overline{\triangle t}}$, i.e., $\overline{\triangle t}=-\frac{1}{\tau_{2}} \ln \left(\frac{a}{b}\right)$. For all $\triangle t<\overline{\triangle t}$ exist $\varepsilon>0$ and $\eta \geq 0$ such that $\delta_{1} c_{1}(a+\varepsilon)=v_{\alpha}(b-\eta)$. Since $a+\varepsilon$ is by construction of $v$ a fixed point of $\delta_{2} v^{-1}\left(\delta_{1} v\right)$ it follows that $a+\varepsilon$ is a fixed point of $\delta_{2} c_{2}\left(\delta_{1} c_{1}\right)$ if $\triangle t<\overline{\triangle t}$. Therefore, for all $\Delta t<\overline{\triangle t}-\widetilde{\varepsilon}$ with $\widetilde{\varepsilon} \in(0, \overline{\triangle t})$, the function $\delta_{2} c_{2}\left(\delta_{1} c_{1}(p)\right)$ has a continuum of fixed points in the interval $(a, b)$. Let $p_{f 1}$ and $p_{f 2}$ denote two fixed points of $\delta_{2} c_{2}\left(\delta_{1} c_{1}\right)$ with $p_{f 1} \neq p_{f 2}$. For $\triangle t<\overline{\triangle t}-\widetilde{\varepsilon}$ there exists a continuum of SPNE where each SPNE corresponds to a fixed point. Note that $\overline{\triangle t}>0$ if $a<b$. As $\Delta t \rightarrow 0$, the minimum lenght of $I$ such that a continuum of fixed points exists, goes to zero. Hence, as $\triangle t \rightarrow 0$, a continuum of equilibria exist whenever $a<b$, i.e., whenever $c_{1}\left(p_{2}\right)^{-\rho} p_{2}$ is quasiconcave but not strictly quasiconcave. 
Case 2: There exists an interval $A$ such that $\forall v_{\alpha}$ with $\alpha \in A$ exist at least two disjoint intervals $I_{1}$ and $I_{2}$ such that $c_{1}(p) \geq v_{\alpha}(p)$ if and only if $p \in \bigcup I$.

For an arbitrary $\widetilde{\alpha} \in A$, let $a_{1}=\inf I_{1}$ and $b_{1}=\sup I_{1}$ and define $a_{2}$ and $b_{2}$ analogously. Let $\overline{\triangle t}_{1}=-\frac{1}{\tau_{2}} \ln \left(\frac{a_{1}}{b_{1}}\right)$ and $\overline{\triangle t}_{2}=-\frac{1}{\tau_{2}} \ln \left(\frac{a_{2}}{b_{2}}\right)$ and let $\overline{\triangle t}=\min \left\{\overline{\triangle t}_{1}, \overline{\triangle t}_{2}\right\}$. Similar to the argument in part (i), for $\triangle t \leq \overline{\triangle t}$ and corresponding $\delta_{1}, \delta_{2}$ exist $\alpha_{1}, \alpha_{2} \leq \widetilde{\alpha}$ and $\left(p_{a 1}, p_{b 1}\right) \in$ $\left[a_{1}, b_{1}\right]^{2}$ with $p_{a 1}=\delta_{2} p_{b 1}$ such that $c_{1}\left(p_{a 1}\right)=v_{\alpha_{1}}\left(p_{a 1}\right)$ and $c_{1}\left(p_{b 1}\right) \geq v_{\alpha_{1}}\left(p_{b 1}\right) \geq c_{1}^{+}\left(p_{b 1}\right)$ and similar for $p_{a 2}, p_{b 2}$. By the same argument as in part (i), there exist $p_{f 1} \in\left[a_{1}, b_{1}\right)$ and $p_{f 2} \in\left[a_{2}, b_{2}\right)$ such that $p_{f 1}$ and $p_{f 2}$ are fixed points of $\delta_{2} c_{2}\left(\delta_{1} c_{1}\right)$. Whenever $c_{1}\left(p_{2}\right)^{-\rho} p_{2}$ is not quasiconcave, there exists $\widetilde{\alpha}>0$ and intervals $I_{1}$ and $I_{2}$ such that $\overline{\Delta t}>0$. Hence, in the limit as $\triangle t \rightarrow 0$, at least two fixed points $p_{f 1}$ and $p_{f 2}$ exist. Therefore, whenever $c_{1}\left(p_{2}\right)^{-\rho} p_{2}$ is not quasiconcave, at least two SPNE exist: player 1 offers $p_{f 1}\left(p_{f 2}\right)$ and rejects any offer less than $\delta_{1} c_{1}\left(p_{f 1}\right)\left(\delta_{1} c_{1}\left(p_{f 2}\right)\right)$ and accepts otherwise. Player 2 offers $\delta_{1} c_{1}\left(p_{f 1}\right) \quad\left(\delta_{1} c_{1}\left(p_{f 2}\right)\right)$ and rejects any offer less than $p_{f 1}\left(p_{f 2}\right)$ and accepts otherwise.

The following technical Lemma about $\rho$-concave functions is needed to prove Theorem 2.

Lemma $\mathbf{A 1}$ (i) Let $x_{1} \neq x_{2}$ and $y_{1}, y_{2}>0$ be given. For all $\rho \neq 0,|\rho|<\infty$, there exists a unique exactly $\rho$-concave function $g$ with $g(x)=(d x+k)^{\frac{1}{\rho}}$ such that $g\left(x_{1}\right)=y_{1}$ and $g\left(x_{2}\right)=y_{2}$.

(ii) If $g$ is exactly $\rho$-concave and $g$ is not constant, then the inverse $g^{-1}$ exists. If $g$ is exactly $\rho$-concave with $g(x)=(d x+k)^{\frac{1}{\rho}}$ and $\rho=-\frac{\ln \delta_{2}}{\ln \delta 1}$ and inverse $g^{-1}$, then $\delta_{2} g^{-1}\left(\delta_{1} g(x)\right)=$ $x+\frac{k}{d}\left(1-\delta_{2}\right)$.

(iii) Let $\rho^{\prime}<\rho$. If $f$ is $\rho$-concave, $f$ is also $\rho^{\prime}$-concave. If $g$ is exactly $\rho$-concave and $f$ is strictly $\rho$-concave and if there exists $x_{1}, x_{2}$ with $x_{1}<x_{2}$ and $g\left(x_{1}\right)=f\left(x_{1}\right)$ and $g\left(x_{2}\right)=f\left(x_{2}\right)$, then $g(x)<f(x) \forall x \in\left(x_{1}, x_{2}\right)$ and $g(x)>f(x) \forall x \notin\left[x_{1}, x_{2}\right]$.

(iv) If $g_{1}$ and $g_{2}$ are exactly $\rho$-concave and if there exists $x_{1}<x_{1}$ such that $g_{1}\left(x_{1}\right)=g_{2}\left(x_{1}\right)$ and $g_{1}\left(x_{2}\right)<g_{2}\left(x_{2}\right)$, then $g_{1}(x)>g_{2}(x) \forall x<x_{1}$ and $g_{1}(x)<g_{2}(x) \forall x>x_{1}$.

(v) Let $g_{1}$ and $g_{2}$ be exactly $\rho$-concave and strictly decreasing functions with $\rho<0$ and $g_{i}=$ 
$\left(d_{i} x+k_{i}\right)^{\frac{1}{\rho}}$ for $i \in\{1,2\}$. If there exists $x_{r}<x_{c}$ such that $g_{1}\left(x_{c}\right)=g_{2}\left(x_{c}\right)$ and $g_{1}\left(x_{r}\right)<g_{2}\left(x_{r}\right)$, then there exists $\triangle>0$ such that $\frac{k_{1}}{d_{1}}>\frac{k_{2}}{d_{2}}+\triangle$.

Note that if $g$ is exactly (strictly) $\rho$-concave, then $g^{\rho}$ is affine (strictly concave).

Theorem 2 ( $\triangle t$ not constant) Let $P \in P_{\rho} \forall \rho \in[\underline{\rho}, \bar{\rho}]$ and suppose there exists $\varepsilon>0$ such that $c_{1}$ is strictly $\bar{\rho}$-concave for all $p \in\left[\underline{p}_{2}^{N}-\varepsilon, \bar{p}_{2}^{N}+\varepsilon\right]$. Then there exists a unique strategy profile that survives iterated conditional dominance.

Proof. Similar to the proof of Theorem 1(i), I show that $\left(r_{2}^{n}\right)$ and $\left(a_{2}^{n}\right)$ converge to the same limit. As shown in the proof of Theorem 1(i), whenever $P \in P_{\rho}$, the function $\delta_{2} c_{2}\left(\delta_{1} c_{1}\right)$ has a unique fixed point $p_{f} \in\left[\delta_{2} p_{2}^{N}, p_{2}^{N}\right)$. Note that $p_{f}$ is a function of the discount factors. Since $p_{f} \in\left[\delta_{2} p_{2}^{N}, p_{2}^{N}\right)$ and since $P \in P_{\rho} \forall \rho \in[\underline{\rho}, \bar{\rho}]$, for all $t$ even, the fixed point of $\delta_{2}^{t} c_{2}\left(\delta_{1}^{t+1} c_{1}\right)$ lies in the interval $\left[\underline{p}_{2}^{N}, \bar{p}_{2}^{N}\right)$. Furthermore, as shown in the proof of Theorem $1(\mathrm{i}), \delta_{2} c_{2}\left(\delta_{1} c_{1}(p)\right)>p$ for $0 \leq p<p_{f}$ and $\delta_{2} c_{2}\left(\delta_{1} c_{1}(p)\right)<p$ for $p_{f}<p \leq \bar{p}_{2}$. Since $P \in P_{\rho} \forall \rho \in[\underline{\rho}, \bar{\rho}]$, for every $\varepsilon>0$ there exists an $n$ such that $r_{2}^{n} \in\left[\underline{p}_{2}^{N}-\varepsilon, \bar{p}_{2}^{N}+\varepsilon\right]$ and $a_{2}^{n} \in\left[\underline{p}_{2}^{N}-\varepsilon, \bar{p}_{2}^{N}+\varepsilon\right]$. Theorem 2 requires that there exists $\varepsilon>0$ such that $c_{1}$ is strictly $\bar{\rho}$-concave for all $p \in\left[\underline{p}_{2}^{N}-\varepsilon, \bar{p}_{2}^{N}+\varepsilon\right]$. With a slight abuse of notation, let $I_{\varepsilon}$ be the largest interval $\left[\underline{p}_{2}^{N}-\varepsilon, \bar{p}_{2}^{N}+\varepsilon\right]$ such that $c_{1}$ is strictly $\bar{\rho}$-concave for all $p \in I_{\varepsilon}$. Since there exists $n$ such that $r_{2}^{n} \in I_{\varepsilon}$ and $a_{2}^{n} \in I_{\varepsilon}$, it is sufficient to show that the sequences $\left(r_{2}^{n}\right)$ and $\left(a_{2}^{n}\right)$ converge to the same limit given that $r_{2}^{n} \in I_{\varepsilon}$ and $a_{2}^{n} \in I_{\varepsilon}$. Hence, for rest of the proof, I restrict attention to the case where $r_{2}^{n} \in I_{\varepsilon}$ and $a_{2}^{n} \in I_{\varepsilon}$.

Recall that $r_{2}^{n+1}=\delta_{2}^{2} c_{2}\left(\delta_{1}^{3} c_{1}\left(\delta_{2}^{4} c_{2}\left(\delta_{1}^{5} c_{1}\left(r_{2}^{n-2}\right)\right)\right)\right)$ and so on, and that $\rho=-\frac{\ln \delta_{2}^{t}}{\ln \delta_{1}^{t+1}}$. Therefore, we need to show that for all $\delta_{2}^{t}, \delta_{1}^{t+1}$ with $t$ even and $\rho \in[\underline{\rho}, \bar{\rho}]$ we have $a_{2}^{n+1}-r_{2}^{n+1}<a_{2}^{n}-r_{2}^{n}$ where $a_{2}^{n+1}=\delta_{2}^{t} c_{2}\left(\delta_{1}^{t+1} c_{1}\left(a_{2}^{n}\right)\right)$ and similar for $r_{2}^{n+1}$. To simplify notation, I suppress the time indices of the discount factors. The parameter $\rho$ corresponds to the $\delta_{2}^{t}, \delta_{1}^{t+1}$ under consideration.

As shown in the proof of Lemma $1, a_{i}^{n} \geq r_{i}^{k} \forall n, k$. If $r_{2}^{n}=a_{2}^{n}$ then $a_{2}^{\infty}=r_{2}^{\infty}$ and we are done. If $r_{2}^{n}=p_{f}$ and $a_{2}^{n}>p_{f}$, then $r_{2}^{n+1}=r_{2}^{n}$ and $a_{2}^{n+1}<a_{2}^{n}$ (where $a_{2}^{n+1}<a_{2}^{n}$ follows from 
$\delta_{2} c_{2}\left(\delta_{1} c_{1}(0)\right)>0$, and $\delta_{2} c_{2}\left(\delta_{1} c_{1}(\bar{p})\right) \leq \delta_{2} \bar{p}_{2}$, and the fact that $\delta_{2} c_{2}\left(\delta_{1} c_{1}\right)$ is increasing and has a unique fixed point). Hence, if $r_{2}^{n}=p_{f}$ and $a_{2}^{n}>p_{f}$, we have $a_{2}^{n+1}-r_{2}^{n+1}<a_{2}^{n}-r_{2}^{n}$. Similarly, if $a_{2}^{n}=p_{f}$ and $r_{2}^{n}<p_{f}$, we have $r_{2}^{n+1}>r_{2}^{n}$ and $a_{2}^{n+1}-r_{2}^{n+1}<a_{2}^{n}-r_{2}^{n}$. Note that $p_{f}$ depends on the discount factors. Given some $r_{2}^{n}, a_{2}^{n}$ it is possible that there exists some $t$ and $\delta_{2}^{t}, \delta_{1}^{t+1}$ and corresponding fixed point $p_{f}$ such that $r_{2}^{n}<a_{2}^{n}<p_{f}$ or $p_{f}<r_{2}^{n}<a_{2}^{n}$. Therefore, it remains to show that $a_{2}^{n+1}-r_{2}^{n+1}<a_{2}^{n}-r_{2}^{n}$ if $r_{2}^{n} \in I_{\varepsilon}, a_{2}^{n} \in I_{\varepsilon}$ and if $r_{2}^{n}<a_{2}^{n}<p_{f}$ (Case I) or $p_{f}<r_{2}^{n}<a_{2}^{n}$ (Case II).

For $r_{2}^{n}, a_{2}^{n} \in I_{\varepsilon}$ follows from Lemma A1(i) that there exist unique $d_{r}$ and $k_{r}$ that define an exact $\rho$-concave function $g_{r}$ with $g_{r}(p)=\left(d_{r} p+k_{r}\right)^{\frac{1}{\rho}}$ such that $g_{r}\left(r_{2}^{n}\right)=c_{1}\left(r_{2}^{n}\right)$ and $g_{r}\left(\frac{r_{2}^{n+1}}{\delta_{2}}\right)=$ $c_{1}\left(\frac{r_{2}^{n+1}}{\delta_{2}}\right)$. Define $g_{a}$ in the same way such that $g_{a}$ intersects $c_{1}$ at $a_{2}^{n}$ and $\frac{a_{2}^{n+1}}{\delta_{2}}$. Since $c_{1}$ is weakly decreasing and strictly $\bar{\rho}$-concave for all $p \in I_{\varepsilon}, c_{1}$ is continuous and strictly decreasing for all $p \in I_{\varepsilon}$. Therefore, for $p \in I_{\varepsilon}, c_{2}$ is the inverse of $c_{1}$ and $c_{2}$ is strictly decreasing for $p \in\left[c_{1}\left(\min I_{\varepsilon}\right), c_{1}\left(\max I_{\varepsilon}\right)\right]$. Since $r_{2}^{n} \in I_{\varepsilon}$ we have $c_{2}\left(c_{1}\left(r_{2}^{n}\right)\right)=r_{2}^{n}$ and $c_{2}\left(\delta_{1} c_{1}\left(r_{2}^{n}\right)\right)>r_{2}^{n}$. From $r_{2}^{n+1}=\delta_{2} c_{2}\left(\delta_{1} c_{1}\left(r_{2}^{n}\right)\right)$ follows that $r_{2}^{n}<\frac{r_{2}^{n+1}}{\delta_{2}}$ and, similarly, $a_{2}^{n}<\frac{a_{2}^{n+1}}{\delta_{2}}$. Since $c_{1}$ is strictly decreasing for all $p \in I_{\varepsilon}$ and $c_{1}\left(p_{2}\right)^{-\rho} p_{2}$ is strictly quasiconcave and $\bar{p}_{2}^{N}>\arg \max c_{1}\left(p_{2}\right)^{-\rho} p_{2}$, we have $c_{1}$ strictly decreasing for all $p \geq \min I_{\varepsilon}$. Hence $r_{2}^{n}<\frac{r_{2}^{n+1}}{\delta_{2}}$ implies that $g_{r}\left(r_{2}^{n}\right)>g_{r}\left(\frac{r_{2}^{n+1}}{\delta_{2}}\right)$ and, similarly, $g_{a}\left(a_{2}^{n}\right)>g_{a}\left(\frac{a_{2}^{n+1}}{\delta_{2}}\right)$. Since $r_{2}^{n}<a_{2}^{n}$, we have $\frac{r_{2}^{n+1}}{\delta_{2}}<\frac{a_{2}^{n+1}}{\delta_{2}}$. Since $c_{1}$ is strictly decreasing for $p \geq \min I_{\varepsilon}$, we know that $g_{r}$ and $g_{a}$ are strictly decreasing. Since $g(p)=(d p+k)^{\frac{1}{\rho}}$ with $\rho<0$, this implies that $d_{r}>0$ and $d_{a}>0$. Since $r_{2}^{n}<\frac{r_{2}^{n+1}}{\delta_{2}}$, from Lemma A1(iii) follows that $g_{r}(p)<c_{1}(p) \forall p \in\left(r_{2}^{n}, \frac{r_{2}^{n+1}}{\delta_{2}}\right)$ and that $g_{r}(p)>c_{1}(p) \forall p \notin\left[r_{2}^{n}, \frac{r_{2}^{n+1}}{\delta_{2}}\right]$ and similar for $g_{a}$. Since $r_{2}^{n}<a_{2}^{n}$ we have $g_{a}\left(r_{2}^{n}\right)>c_{1}\left(r_{2}^{n}\right)$ and, therefore, $g_{a}\left(r_{2}^{n}\right)>g_{r}\left(r_{2}^{n}\right)$. Similarly, since $\frac{r_{2}^{n+1}}{\delta_{2}}<\frac{a_{2}^{n+1}}{\delta_{2}}$ we have $g_{r}\left(\frac{a_{2}^{n+1}}{\delta_{2}}\right)>g_{a}\left(\frac{a_{2}^{n+1}}{\delta_{2}}\right)$. Since $g$ is exactly $\rho$-concave, $g$ is continuous. Therefore, there exists a unique $p_{c} \in\left(r_{2}^{n}, \frac{a_{2}^{n+1}}{\delta_{2}}\right)$ such that $g_{r}\left(p_{c}\right)=g_{a}\left(p_{c}\right)$. Hence, $g_{r}(p)<g_{a}(p)$ for all $p \leq r_{2}^{n}$ and $g_{r}(p)>g_{a}(p)$ for all $p \geq \frac{a_{2}^{n+1}}{\delta_{2}}$.

Case I: $r_{2}^{n}<a_{2}^{n}<p_{f}$

As shown in the proof of Theorem 1(i), for a fixed point $p_{f}$ of $\delta_{2} c_{2}\left(\delta_{1} c_{1}\right)$ there exists $\alpha_{f}$ such 
that $v_{\alpha_{f}}\left(p_{f}\right)=c_{1}\left(p_{f}\right)$ and $c_{1}\left(\frac{p_{f}}{\delta_{2}}\right) \geq v_{\alpha_{f}}\left(\frac{p_{f}}{\delta_{2}}\right) \geq c_{1}^{+}\left(\frac{p_{f}}{\delta_{2}}\right)$. Define $\alpha_{a}$ such that $v_{\alpha_{a}}\left(a_{2}^{n}\right)=g_{a}\left(a_{2}^{n}\right)$ where $g_{a}\left(a_{2}^{n}\right)=c_{1}\left(a_{2}^{n}\right)$. Recall that $\widehat{p}=\arg \max c_{1}\left(p_{2}\right)^{-\rho} p_{2}$. Note that $c_{1}\left(p_{2}\right)^{-\rho} p_{2}$ is strictly quasiconcave, that $v_{\alpha}(p)^{-\rho} \cdot p=\alpha$, that (by assumption) $a_{2}^{n}<p_{f}$, and (as shown in proof of Theorem 1(i)) that $p_{f}<\widehat{p}$. Hence $c_{1}\left(a_{2}^{n}\right)^{-\rho} \cdot a_{2}^{n}<c_{1}\left(p_{f}\right)^{-\rho} \cdot p_{f}$. Hence, there exists a unique $\widetilde{p}>\frac{p_{f}}{\delta_{2}}$ such that $c_{1}(\widetilde{p}) \geq v_{\alpha_{a}}(\widetilde{p}) \geq c_{1}^{+}(\widetilde{p})$. Since $a_{2}^{n}<p_{f}$ we have $\frac{a_{2}^{n+1}}{\delta_{2}}<\frac{p_{f}}{\delta_{2}}$ and, therefore, $\frac{a_{2}^{n+1}}{\delta_{2}}<\widetilde{p}$. From Lemma A1(iii) follows that $g_{a}(\widetilde{p})>c_{i}(\widetilde{p})$ and, therefore, $g_{a}(\widetilde{p})>v_{\alpha_{a}}(\widetilde{p})$. From Lemma A1(iv) follows that $g_{a}(p)>v_{\alpha_{a}}(p) \forall p>a_{2}^{n}$ and $g_{a}(p)<v_{\alpha_{a}}(p) \forall p<a_{2}^{n}$. Recall that $v_{\alpha_{a}}(p)=\left(\frac{p}{\alpha_{a}}\right)^{\frac{1}{\rho}}$ which can be written as $v_{\alpha_{a}}(p)=\left(d_{v} p+k_{v}\right)^{\frac{1}{\rho}}$ where $k_{v}=0$. Applying Lemma $\mathrm{A} 1(\mathrm{v})$ where $g_{a}$ corresponds to $g_{1}$ and $v_{\alpha_{a}}$ corresponds to $g_{2}$ gives $\frac{k_{a}}{d_{a}}>0$.

Case II: $p_{f}<r_{2}^{n}<a_{2}^{n}$

The argument is similar to Case I. Define $v_{\alpha_{r}}$ such that $v_{\alpha_{r}}\left(r_{2}^{n}\right)=g_{r}\left(r_{2}^{n}\right)$ where $g_{r}\left(r_{2}^{n}\right)=c_{1}\left(r_{2}^{n}\right)$. If $r_{2}^{n}<\widehat{p}$, there exists $\widetilde{p} \in\left(\widehat{p}, \frac{p_{f}}{\delta_{2}}\right)$ such that $c_{1}(\widetilde{p}) \geq v_{\alpha_{r}}(\widetilde{p}) \geq c_{1}^{+}(\widetilde{p})$. Since $\frac{r_{2}^{n+1}}{\delta_{2}}>\frac{p_{f}}{\delta_{2}}$, it follows from Lemma A1(iii) that $c_{1}\left(\frac{r_{2}^{n+1}}{\delta_{2}}\right)<v_{\alpha_{r}}\left(\frac{r_{2}^{n+1}}{\delta_{2}}\right)$ and, therefore, $g_{r}\left(\frac{r_{2}^{n+1}}{\delta_{2}}\right)<v_{\alpha_{r}}\left(\frac{r_{2}^{n+1}}{\delta_{2}}\right)$. From Lemma A1(iv) follows that $g_{r}(p)<v_{\alpha_{r}}(p) \forall p>r_{2}^{n}$ and $g_{r}(p)>v_{\alpha_{r}}(p) \forall p<r_{2}^{n}$. Similar, if $r_{2}^{n}>$ $\widehat{p}$, there exists $\widetilde{p}<\widehat{p}$ such that $c_{1}(\widetilde{p})=v_{\alpha_{r}}(\widetilde{p})$. From Lemma A1(iii) follows that $g_{r}(\widetilde{p})>v_{\alpha_{r}}(\widetilde{p})$ and, therefore, $g_{r}(p)<v_{\alpha_{r}}(p) \forall p>r_{2}^{n}$ and $g_{r}(p)>v_{\alpha_{r}}(p) \forall p<r_{2}^{n}$. If $r_{2}^{n}=\widehat{p}$, there exists no $\widetilde{p} \neq \widehat{p}$ such that $c_{1}(\widetilde{p})=v_{\alpha_{r}}(\widetilde{p})$. Hence, $c_{1}(p)<v_{\alpha_{r}}(p) \forall p \neq \widehat{p}$. Hence, $g_{r}\left(\frac{r_{2}^{n+1}}{\delta_{2}}\right)<v_{\alpha_{r}}\left(\frac{r_{2}^{n+1}}{\delta_{2}}\right)$ and $g_{r}(p)<v_{\alpha_{r}}(p) \forall p>r_{2}^{n}$ and $g_{r}(p)>v_{\alpha_{r}}(p) \forall p<r_{2}^{n}$. Applying Lemma A1(v) where $g_{r}$ corresponds to $g_{1}$ and $v_{\alpha_{a}}$ corresponds to $g_{2}$ gives $\frac{k_{r}}{d_{r}}<0$.

Applying Lemma A1(v) where $g_{r}$ corresponds to $g_{1}$ and $g_{a}$ corresponds to $g_{2}$ shows that whenever $a_{2}^{n}-r_{2}^{n}>0$ there exists $\triangle>0$ such that $\frac{k_{r}}{d_{r}}>\frac{k_{a}}{d_{a}}+\triangle$. From Lemma A1(ii) and the definition of $g_{r}$ follows that $r_{2}^{n+1}=\delta_{2} g_{r}^{-1}\left(\delta_{1} g_{r}\left(r_{2}^{n}\right)\right)=r_{2}^{n}+\frac{k_{r}}{d_{r}}\left(1-\delta_{2}\right)$ and analogue for $a_{2}^{n+1}$. Hence, whenever $r_{2}^{n} \in I_{\varepsilon}$ and $a_{2}^{n} \in I_{\varepsilon}$ we have $a_{2}^{n+1}-r_{2}^{n+1}<a_{2}^{n}-r_{2}^{n}-\triangle\left(1-\delta_{2}\right)$. As shown above, there exists $n$ such that $r_{2}^{n} \in I_{\varepsilon}, a_{2}^{n} \in I_{\varepsilon}$. Therefore, $\left(a_{2}^{n}\right)$ and $\left(r_{2}^{n}\right)$ converge to the same limit. Let $p_{2}^{\infty}$ denote the limit, i.e., $p_{2}^{\infty}=r_{2}^{\infty}=a_{2}^{\infty}$.

Since both $\left(a_{2}^{n}\right)$ and $\left(r_{2}^{n}\right)$ converge to $p_{2}^{\infty}$, it follows from Lemma 1 that it is conditionally dom- 
inated for player 2 to reject (accept) an offer with $p_{2}>p_{2}^{\infty}\left(p_{2}<p_{2}^{\infty}\right)$. Hence, it is conditionally dominated for player 1 to accept (reject) an offer where $p_{1}<\delta_{1} c_{1}\left(p_{2}^{\infty}\right)\left(p_{1}>\delta_{1} c_{1}\left(p_{2}^{\infty}\right)\right)$. Since $c_{1}$ is strictly $\bar{\rho}$-concave for all $p \in\left[\underline{p}_{2}^{N}-\varepsilon, \bar{p}_{2}^{N}+\varepsilon\right]$, we have $c_{1}^{\prime}\left(p_{2}^{\infty}\right)<0$. Hence, it is conditionally dominated for player 2 to offer $p_{1} \neq \delta_{1} c_{1}\left(p_{f}\right)$ and it is conditionally dominated for player 1 to offer $p_{2} \neq p_{2}^{\infty}$. Therefore, a unique strategy profile survives iterated conditional dominance.

Lemma 2 If $\triangle t$ is constant and $P^{s} \in P_{\rho}$, then $f(\Gamma(P, \gamma, b))=f\left(\Gamma\left(P^{s}, 0,0\right)\right)+s$. For $\triangle t$ not constant, if $P^{s} \in P_{\rho} \forall \rho \in[\underline{\rho}, \bar{\rho}]$ and if there exists $\varepsilon>0$ such that $c_{1}$ is strictly $\bar{\rho}$-concave for all $p \in\left[\underline{p}_{2}^{N}-\varepsilon, \bar{p}_{2}^{N}+\varepsilon\right]$, then $f(\Gamma(P, \gamma, b))=f\left(\Gamma\left(P^{s}, 0,0\right)\right)+s$.

Proof. The relation $\delta_{i}^{t} c_{i}\left(\delta_{j}^{t+1} c_{j}\left(p_{i}\right)+\gamma_{j}^{t+1}+b_{j}^{t+1}\right)+\gamma_{i}^{t}+b_{i<}^{t>} p_{i}$ describes the iteration process for $\Gamma(P, \gamma, b)$. Note that $b_{i}^{t}$ and $\gamma_{i}^{t}$ depend on the delays, i.e. on $H_{t}$. Rewrite $b_{i}^{t}$ as $\frac{-\ln \mu \cdot b_{i}}{\tau_{i}-\ln \mu} \int_{0}^{\infty}(1-$ $\left.\mu^{t} e^{-\tau_{i} t}\right) h(t) d t$ and $\gamma_{i}^{t}$ as $\frac{\gamma_{i}}{\tau_{i}-\ln \mu}\left(1+\left[\mu^{t} e^{-\tau_{i} t} H(t)\right]_{0}^{\infty}-\int_{0}^{\infty} \mu^{t} e^{-\tau_{i} t} h(t) d t\right)$. Then $\frac{\gamma_{i}^{t}+b_{i}^{t}}{1-\delta_{i}^{t}}=\frac{\gamma_{i}-\ln \mu \cdot b_{i}}{\tau_{i}-\ln \mu}=$ $s_{i} \forall t$. Substituiting in the relation above gives $\delta_{i}^{t} c_{i}\left(\delta_{j}^{t+1} c_{j}\left(p_{i}\right)-\delta_{j}^{t+1} s_{j}+s_{j}\right)-\delta_{i}^{t} s_{i}{ }_{<} p_{i}-s_{i}$. Let $u$ denote the rescaled value, $u_{i}=p_{i}-s_{i}$. Then the relation can be written as

$$
\delta_{i}^{t}\left(c_{i}\left(\delta_{j}^{t+1}\left[c_{j}\left(u_{i}+s_{i}\right)-s_{j}\right]+s_{j}\right)-s_{i}\right) \gtrless u_{i}
$$

which is the same as $\delta_{i}^{t} c_{i}^{s}\left(\delta_{j}^{t+1} c_{j}^{s}(u)\right)_{<}^{>} u$. The limit of $\delta_{i}^{t} c_{i}\left(\delta_{j}^{t+1} c_{j}(\ldots)+\gamma_{j}^{t+1}+b_{j}^{t+1}\right)+\gamma_{i}^{t}+b_{i}^{t}$ is the same as the limit of $\delta_{i}^{t} c_{i}^{s}\left(\delta_{j}^{t+1} c_{j}^{s}(\ldots)\right)+s_{i}$. A payoff $u$ in $\Gamma\left(P^{s}, 0,0\right)$ corresponds to payoff of $p$ in $\Gamma(P, \gamma, b)$ with $p=u+s$. If $P^{s} \in P_{\rho}$ (for $\triangle t$ constant) or $P^{s} \in P \rho \forall \rho \in[\underline{\rho}, \bar{\rho}]$ and $c_{1}^{s}$ is strictly $\bar{\rho}$-concave for all $p \in I_{\varepsilon}$ (if $\triangle t$ not constant), then $\left(r_{i}^{n}\right)$ and $\left(a_{i}^{n}\right)$ of $\Gamma\left(P^{s}, 0,0\right)$ converge to the same limit (Theorem 1 and Theorem 2, respectively). Hence, $\left(r_{i}^{n}\right)$ and $\left(a_{i}^{n}\right)$ of $\Gamma(P, \gamma, b)$ converge to the same limit and the limits $r_{i}^{\infty}=a_{i}^{\infty}$ of $\Gamma(P, \gamma, b)$ and $\Gamma\left(P^{s}, 0,0\right)$ differ by $s_{i}$. Hence, the set of strategies that survive iterated conditional dominance differs by the constant $s$. 


\section{References}

[1] Binmore K (1987) Nash Bargaining Theory II. In: Binmore K, Dasgupta P (eds.) The Economics of Bargaining. Blackwell, Oxford, pp. 61-76

[2] Binmore K, Rubinstein A and Wolinsky A (1986) The Nash Bargaining Solution in Economic Modeling. Rand Journal of Economics 17: 176-188

[3] Conley JP, Wilkie S (1996) An Extension of the Nash Bargaining Solution to Nonconvex Problems. Games and Economic Behavior 13: 26-38

[4] Conley JP, Wilkie S (1995) Implementing the Nash extension bargaining solution for non-convex problems. Economic Design 1: 205-216, revised mimeo 2000

[5] Herrero M (1989) The Nash-Program: Non-convex Bargaining Problems. Journal of Economic Theory 49: 266-277

[6] Lopomo G, Ok EA (2001) Bargaining, interdependence, and the rationality of fair division. RAND Journal of Economics 32(2): 263-283

[7] Muthoo A (1999) Bargaining Theory with Applications. Cambridge Univ. Press, Cambridge

[8] Nash J (1950) The Bargaining Problem. Econometrica 28: 155-162

[9] Rubinstein A (1981) Perfect equilibrium in a Bargaining Model. Econometrica 50: 97-109

[10] Shaked A, Sutton J (1984) Involuntary Unemployment as a Perfect Equilibrium in a Bargaining Model. Econometrica 52: 1351-1364 